\title{
La communauté peut aider ses enfants à mieux se préparer à commencer l'école
} L'initiative Comprendre la petite enfance

\section{The Community Can Better Prepare Help Its Children for} School

\author{
Understanding the Early Years Initiative
}

La comunidad puede ayudar a sus niños a estar bien preparados para comenzar la escuela La iniciativa Comprender la infancia

\section{Pierre Lapointe et Isabelle Martin}

\section{Volume 33, numéro 2, automne 2005}

Nouvelles tendances à l'égard de la petite enfance

URI : https://id.erudit.org/iderudit/1079103ar

DOI : https://doi.org/10.7202/1079103ar

Aller au sommaire du numéro

\section{Éditeur(s)}

Association canadienne d'éducation de langue française

ISSN

0849-1089 (imprimé)

1916-8659 (numérique)

Découvrir la revue

Citer cet article

Lapointe, P. \& Martin, I. (2005). La communauté peut aider ses enfants à mieux se préparer à commencer l'école : l'initiative Comprendre la petite enfance. Éducation et francophonie, 33(2), 112-124. https://doi.org/10.7202/1079103ar
Résumé de l'article

L'initiative Comprendre la petite enfance à Montréal s'inscrit dans le cadre du programme national d'aide au développement des enfants et de lutte contre la pauvreté. Toujours en cours, cette étude vise, à partir de trois sources d'information, à rendre compte de l'état du développement des enfants dans certains quartiers de Montréal, au moment de leur entrée à l'école. Les enseignants de maternelle de 28 écoles publiques ont d'abord évalué le degré de préparation scolaire des enfants. Les parents ont fourni des renseignements sur leur développement général. Enfin, les caractéristiques des populations et les ressources disponibles sur le territoire étudié ont été examinées. Les résultats de cette collecte de données sont transmis aux intervenants communautaires pour enrichir les programmes et les ressources offerts aux enfants et aux familles. L'article décrit les origines du projet national, les résultats de l'évaluation de la préparation scolaire des enfants et de celle des caractéristiques socioéconomiques des populations étudiées. On termine en décrivant les activités de transfert des connaissances aux organismes partenaires, dans la perspective d'une révision des plans d'action locaux en petite enfance.
Tous droits réservés (C) Association canadienne d'éducation de langue française, 2005 cest protége par la loi sur le droit d'auteur. L'utilisation des services d'Érudit (y compris la reproduction) est assujettie à sa politique d'utilisation que vous pouvez consulter en ligne. 


\section{La communauté peut aider ses enfants à mieux se préparer à commencer l'école : I'initiative Comprendre la petite enfance}

Pierre LAPOINTE

Université de Montréal, Québec, Canada

\section{Isabelle MARTIN}

Université McGill, Québec, Canada

\section{RÉSUMÉ}

L'initiative Comprendre la petite enfance à Montréal s'inscrit dans le cadre du programme national d'aide au développement des enfants et de lutte contre la pauvreté. Toujours en cours, cette étude vise, à partir de trois sources d'information, à rendre compte de l'état du développement des enfants dans certains quartiers de Montréal, au moment de leur entrée à l'école. Les enseignants de maternelle de 28 écoles publiques ont d'abord évalué le degré de préparation scolaire des enfants. Les parents ont fourni des renseignements sur leur développement général. Enfin, les caractéristiques des populations et les ressources disponibles sur le territoire étudié ont été examinées. Les résultats de cette collecte de données sont transmis aux intervenants communautaires pour enrichir les programmes et les ressources offerts aux enfants et aux familles. L'article décrit les origines du projet national, les résultats de l'évaluation de la préparation scolaire des enfants et de celle des caractéristiques socioéconomiques des populations étudiées. On termine en décrivant les activités de 
transfert des connaissances aux organismes partenaires, dans la perspective d'une révision des plans d'action locaux en petite enfance.

\section{ABSTRACT}

\section{The Community Can Better Prepare Help Its Children for School - Understanding the Early Years Initiative}

Pierre Lapointe

Université de Montréal, Québec, Canada

Isabelle Martin

Université McGill, Québec, Canada

Understanding the Early Years in Montreal is an initiative that is part of the national aid program for child development and the fight against poverty. Still in progress, this study uses three sources of information with the goal of understanding the developmental level of children in some areas of Montréal when they start school. Kindergarten teachers from 28 public schools first evaluated the degree of preparation for school these children had. The parents supplied information on their general development. Finally, the characteristics of the populations and the resources available on the territory being studied were examined. The results of this collection of data were shared with community stakeholders to enrich the programs and the resources available to children and families. The article describes the origins of the national project, the results of the evaluation of these children's preparedness for school and the socio-economic characteristics of the populations being studied. The article concludes with a description of the knowledge transfer activities with partner organizations from the perspective of a revision of local early childhood action plans.

\section{RESUMEN}

La comunidad puede ayudar a sus niños a estar bien preparados para comenzar la escuela: la iniciativa comprender la infancia

Pierre Lapointe

Universidad de Montreal, Quebec, Canadá

Isabelle Martin

Universidad McGill, Quebec, Canadá

La iniciativa Comprender la infancia en Montreal se inscribe en el cuadro del programa nacional de apoyo al desarrollo de los niños y de lucha contra la pobreza. Actualmente en curso, dicho estudio intenta revelar, a partir de tres fuentes de información, el nivel de desarrollo de los niños en ciertas zonas de Montreal, al momento de ingresar a la escuela. Los maestros de las clases de preescolar de 28 escuelas 
públicas evaluaron el grado de preparación escolar de los niños. Los padres de familia proporcionaron las informaciones sobre el desarrollo general de los niños. Finalmente, las características de las poblaciones y los recursos disponibles sobre el territorio estudiado fueron examinadas. Los resultados de ésta recolección de datos fueron transmitidos a los agentes comunitarios para enriquecer los programas y los recursos que se ofrecen a los niños y a las familias. El artículo describe los orígenes del proyecto nacional, los resultados de la evaluación de la preparación escolar de los niños y las características socioeconómicas de las poblaciones estudiadas. Terminamos describiendo las actividades de transferencia de conocimientos hacia los organismos asociados, en una perspectiva de revisión de planes locales de acción entre los infantes.

\section{Introduction}

Notre société doit se donner comme objectif prioritaire d'assurer le développement optimal de chaque enfant afin qu'il réalise son plein potentiel et devienne un citoyen responsable contribuant de manière significative au bien-être de sa collectivité (Conseil des ministres de l'éducation et Statistique Canada, 2003; Développement des ressources humaines Canada et Statistique Canada, 2003).

La réalisation de cet objectif constitue un défi majeur pour les communautés qui ont l'obligation d'adopter des stratégies concertées d'intervention en petite enfance. Ces efforts doivent notamment préparer adéquatement l'enfant à son entrée à l'école. La transition entre la maison et l'école constitue un passage obligé pour tous les enfants. Or, on constate que l'entrée à la maternelle est vécue de manière plus ou moins harmonieuse par les enfants, en raison de facteurs personnels, familiaux et sociaux. Bien que les programmes préscolaires insistent sur l'importance de respecter le rythme d'apprentissage de l'enfant, ses compétences et ses connaissances seront très tôt évaluées selon les standards du système scolaire (Ladd, 1996; Pianta et Cox, 1999; Ramey et Ramey, 1994).

Dans une perspective d'intervention communautaire, la capacité d'agir apparaît souvent limitée parce qu'on n'a pas de données précises sur les enfants, sur les familles et sur les ressources disponibles. La connaissance de l'état du développement des enfants s'avère pourtant une condition nécessaire à l'établissement de relations de concertation et à l'élaboration de stratégies pour assurer une meilleure préparation scolaire des enfants. En collaboration avec certaines communautés, l'initiative Comprendre la petite enfance (CPE) vise à recueillir ces renseignements et à les transmettre aux personnes engagées auprès des enfants et des familles.

L'article donne un aperçu de l'étude, à partir de l'expérience réalisée dans certains quartiers de Montréal. Nous présenterons d'abord les origines de cette initiative nationale, ses assises théoriques, ses objectifs et sa méthodologie. Ensuite, les prin- 
cipaux résultats de l'étude seront traités, notamment ceux concernant l'évaluation de la préparation scolaire des enfants de maternelle et ceux reliés à l'analyse de la vulnérabilité des populations étudiées. Enfin, nous discuterons des stratégies privilégiées pour transférer les connaissances aux acteurs locaux intéressés à revoir leurs plans d'action en petite enfance.

\section{Naissance d'une initiative locale : North York Project}

En 1996, une coalition d'organismes communautaires de la ville de North York (Toronto, Ontario) entreprenait une démarche dont l'objectif était d'implanter une stratégie locale de soutien au développement des enfants. Ce regroupement «The Early Years Action Group » a obtenu du ministère du Développement des ressources humaines du Canada des renseignements sur l'état du développement des enfants de la région étudiée, en vue d'en tracer un portrait et d'élaborer un plan d'action communautaire (Early Years Action Group, 2001). À cette époque, le ministère réalisait, en collaboration avec Statistique Canada, une Étude longitudinale nationale sur les enfants et les jeunes (ELNEJ). Cette recherche pancanadienne, amorcée en 1994, visait à constituer une base de données nationale sur les jeunes Canadiens, de la naissance à l'âge adulte (Développement des ressources humaines du Canada et Statistique Canada, 1996). Le projet de North York offrait l'opportunité aux promoteurs de l'ELNEJ d'expérimenter leur méthodologie de recherche dans une perspective d'intervention régionale. De cette collaboration est née l'initiative Comprendre la petite enfance, qui sera expérimentée dans douze régions du Canada, dont celle de Montréal.

\section{L'initiative Comprendre la petite enfance: un projet national}

À la suite de l'expérimentation à North York, une équipe de recherche du ministère du Développement des ressources humaines du Canada a défini le cadre théorique et méthodologique de l'initiative Comprendre la petite enfance, puis un appel d'offres national a été lancé aux communautés intéressées à y participer. Dans les projets retenus, les objectifs suivants sont poursuivis : établir un portrait de la situation sociale, économique et scolaire des enfants et des familles au moment de l'entrée à l'école des jeunes; diffuser les résultats de la recherche auprès des citoyens et des organismes responsables des services à la petite enfance; et enfin, soutenir la révision des plans d'action locaux en petite enfance.

L'initiative CPE aborde la question de la transition de l'enfant de la maison à l'école en référant au modèle bioécologique de Bronfenbrenner et Morris (1998). Les concepteurs de l'initiative ont recensé les recherches sur les déterminants individuels et familiaux de la préparation scolaire ou "school readiness" (Doherty et Développement des ressources humaines du Canada, 1997). 
Le concept de "school readiness" ou de "maturité scolaire» suscite un grand intérêt aux États-Unis depuis l'adoption des priorités nationales en matière d'éducation, la première stipulant que les jeunes doivent "être prêts à apprendre " au moment de leur entrée à l'école (National Education Goals Panel, 1995). En général, la notion de maturité scolaire indique un ensemble d'habiletés cognitives, affectives et sociales que l'enfant possède, à son entrée à l'école, et qui le rendent plus ou moins bien préparé à la vie scolaire (Rimm-Kaufman et Pianta, 2000).

L'évaluation de la maturité scolaire est un sujet de controverse dans le monde de l'éducation américaine. Plusieurs dénoncent les administrateurs scolaires qui utilisent ces évaluations pour sélectionner les enfants ou leur refuser l'accès à l'école (Kagan et Neuman, 1997; La Paro et Pianta, 2000; May et Kundert, 1997; Stipek et Ryan, 1997). À leur avis, ces approches évaluatives sont centrées exclusivement sur l'enfant et elles négligent les aspects familiaux, scolaires et communautaires qui contribuent à l'acquisition des compétences individuelles. Par contre, ils reconnaissent que ces données peuvent être utiles aux intervenants intéressés à connaître les forces et les faiblesses des enfants, afin de tenir compte de leurs besoins dans l'organisation des services scolaires.

Les concepteurs de l'initiative CPE ont aussi recensé les recherches traitant de l'effet des caractéristiques des quartiers sur le développement de l'enfant (Connors, Brink et Développement des ressources humaines Canada, 1999). Par exemple, les travaux de Kohen, Hertzman, Brooks-Gunn et Développement des ressources humaines du Canada (1998) mettent en évidence la relation entre certaines caractéristiques des quartiers, telles que l'aisance et la cohésion sociale, et les habiletés comportementales et verbales chez les jeunes enfants. Ces chercheurs affirment que la scolarité de la mère et la monoparentalité sont aussi associées à la maturité scolaire de l'enfant et agissent comme facteurs médiateurs des effets du quartier. De même, Wilson (1987) et Brooks-Gunn, Duncan et Aber (1997) illustrent comment l'isolement social et le fait de vivre dans des quartiers défavorisés où les ressources matérielles sont rares, les taux de chômage élevés et les populations peu scolarisées,

Notre étude descriptive exploite différents instruments de mesure pour évaluer les caractéristiques des enfants, de leurs familles et de leur environnement physique et social. peuvent avoir un effet négatif sur le développement des enfants. Dans cette perspective, l'étude des caractéristiques des populations et de leurs quartiers peut aider à mieux cerner les facteurs de risque et de protection qui influent sur ce développement.

À partir d'un devis quasi expérimental de type transversal, notre étude descriptive exploite différents instruments de mesure pour évaluer les caractéristiques des enfants, de leurs familles et de leur environnement physique et social. Les participants sont choisis en fonction de l'école fréquentée par les enfants. L'échantillonnage de plusieurs écoles a délimité un territoire géographique où vivent les enfants. La "communauté " comprend ici les citoyens et les organismes liés aux services d'éducation, aux services bénévoles et récréatifs, aux services de santé, aux services de garde et à l'administration municipale du territoire.

Les douze régions canadiennes partagent une méthodologie de recherche commune. Réalisée durant sept années consécutives, l'initiative CPE prévoit trois phases de collectes de données. 
La communauté peut aider ses enfants à mieux se préparer à commencer l'école:

l'initiative Comprendre la petite enfance

Tableau 1 : Calendrier des activités de la recherche

\begin{tabular}{|lccc|}
\hline Collecte de données & Phase I & Phase II & Phase III \\
\hline Évaluation des enfants par les enseignants & X & X & X \\
\hline Évaluation des enfants par les parents & X & & X \\
\hline Analyse des caractéristiques des populations & $\mathrm{X}$ & $\mathrm{X}$ & $\mathrm{X}$ \\
\hline Évaluation des caractéristiques des quartiers & $\mathrm{X}$ & & $\mathrm{X}$ \\
\hline Inventaire des ressources communautaires & $\mathrm{X}$ & $\mathrm{X}$ \\
\hline Enquête sur les programmes et les services & $\mathrm{X}$ & \\
\hline
\end{tabular}

Au cours de la phase I (2001-2002), un premier groupe d'enfants de maternelle a été évalué par les enseignants dans chaque école du territoire à l'étude. Les données du recensement national ont servi à la constitution d'un indice de vulnérabilité des populations locales (indice social), qui est mis en relation avec la maturité scolaire des enfants. Les parents, quant à eux, fournissent des renseignements sur les caractéristiques de leur enfant et de la famille et sur l'utilisation qu'ils font des ressources et des programmes communautaires. On a complété aussi un inventaire des ressources et des services dédiés aux jeunes enfants et aux familles sur le territoire. De plus, une enquête menée auprès des responsables des services fait connaître la nature et la disponibilité des ressources communautaires. Enfin, la phase I est complétée par une évaluation de l'environnement physique des quartiers couverts par le territoire.

La phase II (2003-2004) consiste à diffuser ces renseignements aux intervenants du milieu, à appuyer les efforts de mobilisation communautaires et à évaluer la maturité scolaire d'une seconde cohorte d'enfants du préscolaire. La phase III (20052007) assure la suite des activités de transfert des connaissances et de mobilisation des communautés. De plus, les enseignants sont invités à évaluer le niveau de préparation scolaire d'une troisième cohorte d'enfants résidant sur le territoire étudié.

\section{L'initiative Comprendre la petite enfance à Montréal}

L'initiative Comprendre la petite enfance à Montréal associe différents partenaires : le Centre 1, 2, 3 Go ! le promoteur, la Commission scolaire de Montréal, la Régie régionale de la santé et des services sociaux de Montréal-Centre, la Ville de Montréal, le Comité de gestion de la taxe scolaire de l'île de Montréal et le Groupe de recherche sur l'inadaptation psychosociale chez l'enfant de l'Université de Montréal.

L'étude est réalisée sur le territoire des centres locaux de services communautaires (CLSC) de Rosemont, Hochelaga-Maisonneuve, Olivier-Guimond, Saint-Michel et Villeray. Les CLSC sont des établissements publics qui ont la responsabilité de rendre accessibles à la population d'un territoire donné des services de santé, des services sociaux, des services de prévention et des services d'action communautaire. Ces ter- 
ritoires comptent environ 300000 habitants et près de $10 \%$ de ce nombre sont des enfants de moins de six ans.

Nous traiterons ici des données sur l'indice social, qui rend compte du niveau de vulnérabilité des populations, et sur l'évaluation de la maturité scolaire. Pour connaître les résultats de l'évaluation des enfants par les parents et les données concernant l'inventaire et l'enquête sur les programmes, le lecteur est invité à consulter les rapports déjà publiés (KSI Research International Inc, 2003; Lapointe, Martin et Robitaille, 2003).

\section{Une mesure de la vulnérabilité des populations : I'indice social}

Neuf indicateurs du Recensement de 1996 ont été sélectionnés pour déterminer le degré de vulnérabilité des populations observées. Selon le découpage géographique établi par Statistique Canada, le territoire étudié se divise en 450 secteurs de dénombrement, qui sont les plus petites unités administratives pour lesquelles des données

Les données montrent la grande vulnérabilité sociale de plusieurs familles sur le territoire étudié. sont disponibles.

Dans la présente analyse, chaque indicateur est reconnu comme un facteur de risque lorsque la condition des individus d'un territoire donné est jugée nettement désavantageuse par rapport à celle observée dans la population canadienne. La somme des facteurs de risque relevés sur un territoire donné constitue la valeur de son indice social. C'est cet indice qui sert à comparer les populations. La valeur de cet indice varie de 0 à 9 : plus elle est élevée, plus la condition socioéconomique de la population est jugée difficile.

Les données montrent la grande vulnérabilité sociale de plusieurs familles sur le territoire étudié, puisque la valeur moyenne de l'indice s'établit à 7 . 
Figure 1: Pourcentage, par école, des enfants jugés en difficulté.

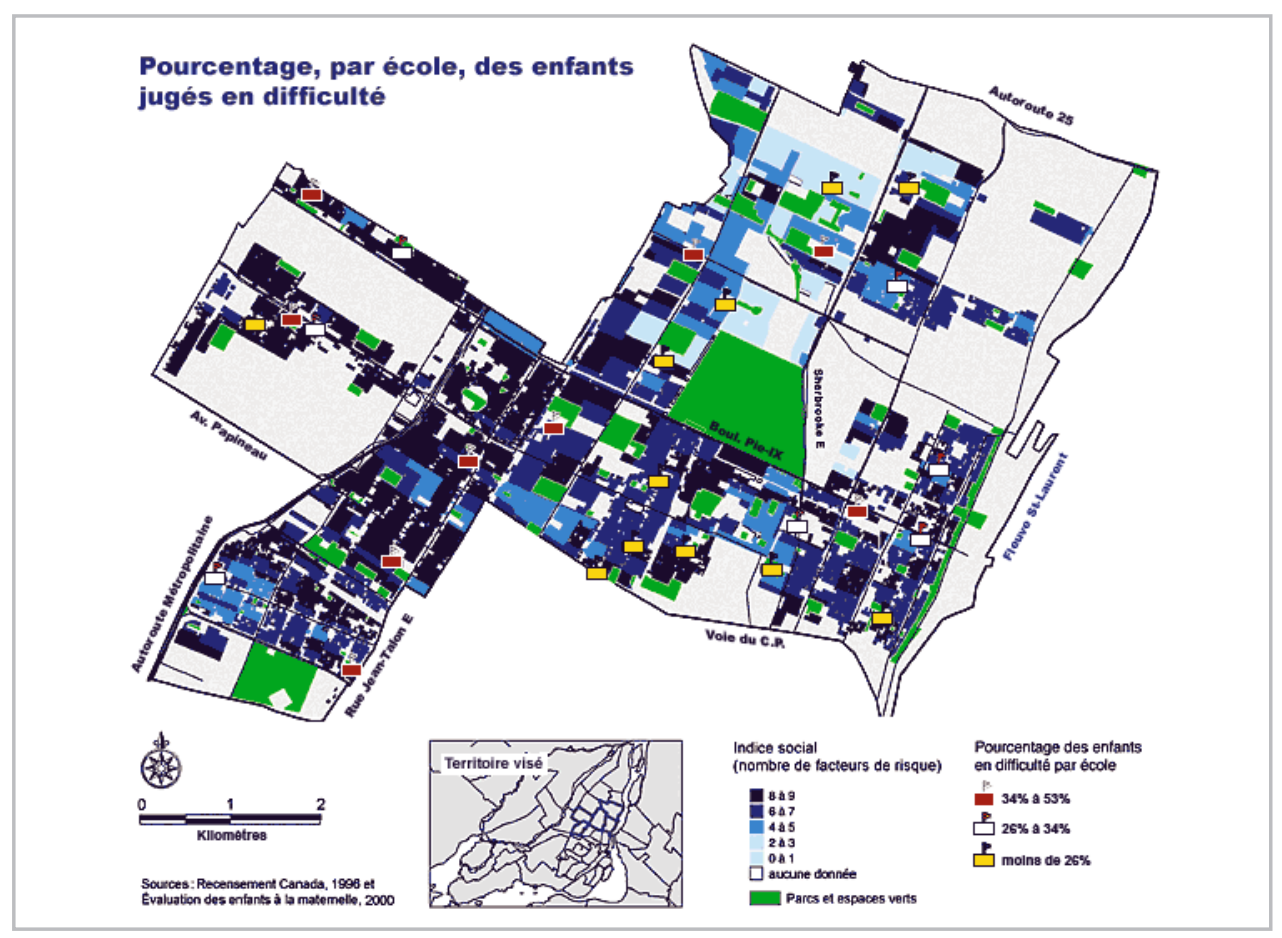

Les enfants et les familles les plus vulnérables habitent des quartiers où il y a plus de ménages à faible revenu; plus d'individus dont une part importante du revenu provient de transferts gouvernementaux; moins de travailleurs actifs; plus de familles monoparentales; un plus grand nombre d'adultes peu scolarisés (sans DES); plus d'immigrants récents; plus de citoyens qui ne parlent ni français ni anglais; moins de ménages propriétaires de leur logement et une plus grande mobilité des familles.

\section{L'évaluation de la maturité scolaire}

L'évaluation de la maturité scolaire des enfants de maternelle fournit des renseignements sur leur développement, au moment de leur entrée à l'école. Au cours de la phase I, dans 28 écoles primaires de la Commission scolaire de Montréal, 70 enseignantes de maternelle ont évalué 1274 enfants à l'aide de l'Instrument de mesure du développement à la petite enfance (Janus et Offord, 2000). Cinq composantes du développement de l'enfant sont évaluées, celles qui sont considérées comme ayant les rapports les plus significatifs avec l'apprentissage et la réussite scolaire: santé physique et bien-être; compétence sociale; maturité affective; développement du langage et aptitudes cognitives; et communication et connaissances générales. 
Dans les écoles du territoire à l'étude, la majorité des enfants obtiennent de bons résultats. Cependant, $32 \%$ d'entre eux éprouvent des difficultés relativement à au moins une composante de la maturité scolaire, tandis que $16 \%$ sont jugés en difficulté à deux composantes et plus. Le pourcentage d'enfants jugés en difficulté est calculé selon le nombre d'enfants dont le résultat individuel relativement à une ou plusieurs composantes de la maturité scolaire se situe au-dessous du $10^{\mathrm{e}}$ rang centile. Les élèves jugés en difficulté à une seule composante n'éprouvent pas nécessairement un problème important de maturité scolaire. Ils peuvent néanmoins être considérés comme des élèves potentiellement à risque. Par contre, les élèves éprouvant des difficultés à deux composantes et plus de la maturité sont les plus susceptibles de devoir affronter des problèmes d'adaptation scolaire.

L'analyse des résultats montre aussi des caractéristiques communes à certains groupes d'enfants. On observe notamment que les filles obtiennent de meilleurs résultats que les garçons à toutes les composantes de la maturité scolaire; les enfants plus âgés semblent mieux préparés que les plus jeunes à affronter les défis scolaires, et les enfants allophones, c'est-à-dire ceux dont la langue maternelle est autre que la langue d'enseignement, obtiennent des résultats plus faibles que les enfants francophones.

Les résultats de l'évaluation de la maturité scolaire, par école cette fois, sont présentés à la figure 1. La proportion d'élèves jugés en difficulté à une ou à plusieurs composantes de la maturité scolaire est indiquée de même que la valeur de l'indice social de chaque secteur. Le taux moyen d'élèves reconnus en difficulté à une composante et plus de la maturité scolaire est de $29 \%$ par école. Le taux minimal est de $6 \%$ alors que le taux maximal est de $53 \%$. Il existe donc, selon l'école d'appartenance, une variation importante du degré de maturité scolaire des enfants. Ces résultats mettent en relief les différences entre les enfants, dès leur entrée à l'école. De plus, on peut observer à la figure 1 la relation entre la préparation scolaire des enfants et la vulnérabilité des populations (indice social). De manière générale, plus la valeur de l'indice social d'un secteur est élevée, c'est-à-dire plus les conditions socioéconomiques sont défavorables, plus le nombre d'enfants jugés en difficulté au regard de la maturité scolaire est grand. Ces analyses illustrent les écarts de performance, dès la maternelle, entre les enfants des milieux défavorisés et ceux des milieux moins défavorisés.

Cette évaluation permet aussi de juger de l'effet du programme d'éducation prématernelle sur la préparation scolaire des enfants à la maternelle. Rappelons que seuls les enfants habitant les quartiers les plus défavorisés de Montréal peuvent aller à l'école à l'âge de quatre ans. Un programme d'activités éducatives en classe et au service de garde est offert dans la plupart des écoles des quartiers défavorisés sur le territoire à l'étude. Deux groupes sont comparés aux fins de l'analyse : le premier est formé d'enfants qui étaient inscrits en classe de prématernelle tandis que le second réunit des sujets qui ne l'étaient pas. Les enfants du premier groupe obtiennent des résultats significativement inférieurs à ceux du second groupe, pour chaque composante de la maturité scolaire. Ainsi, il semble que la participation à un programme de prématernelle ne soit pas une condition suffisante pour que la préparation scolaire 
des enfants des quartiers les plus défavorisés soit équivalente à celle des enfants vivant dans des milieux moins défavorisés.

Une analyse plus détaillée des résultats des enfants de chaque école a été faite au bénéfice des communautés locales et présentée sous la forme d'un Rapport à l'école sur l'évaluation de la maturité des enfants de la maternelle. Un rapport de ce type est remis aux intervenants des écoles participantes de façon qu'ils distinguent mieux les caractéristiques des enfants dont ils ont la charge et adaptent leurs interventions en conséquence.

\section{Le transfert des connaissances}

Dans toutes les régions canadiennes où l'initiative CPE est développée, l'administration des projets est assumée par un organisme reconnu dans la communauté locale pour son engagement dans la promotion du bien-être des enfants et des familles. Le promoteur du projet à Montréal est le Centre 1, 2, 3 GO! et son directeur préside un comité de coordination formé de représentants des partenaires institutionnels de la région. Ces personnes sont informées des résultats de l'étude et elles participent à l'organisation des activités de transfert des connaissances.

En vue d'assurer la diffusion des résultats de la recherche et de soutenir les agents communautaires dans la révision des plans d'action en petite enfance, la principale stratégie adoptée fut celle d'instaurer des collaborations avec des groupes de concertation des services à la petite enfance et à la famille dans les cinq CLSC associés à l'étude. Ces instances locales regroupent des représentants des services de santé, des services sociaux, des services scolaires, des services de garde et des organismes communautaires. De cette manière, nous pouvons joindre simultanément plusieurs types d'intervenants œuvrant sur le territoire.

À l'occasion de ces rencontres d'information et d'échanges, les gens sont invités à former des groupes de réflexion sur la préparation scolaire des enfants dans leurs quartiers. À chaque phase de l'étude, les résultats des dernières évaluations sont discutés avec les partenaires, ce qui maintient leur intérêt et leur collaboration. Parallèlement, les faits saillants de l'étude sont présentés lors de colloques professionnels et communautaires. Enfin, des rapports sont publiés à l'intention des partenaires de l'initiative (KSI Research International Inc, 2003; Lapointe et al., 2003; Lapointe et Robitaille, 2002).

D'emblée, l'étude CPE suscite l'intérêt des intervenants puisqu'elle rend disponibles, dans leur quartier respectif, plusieurs renseignements inédits sur l'état du développement des enfants. Cependant, l'interprétation des données sur la maturité scolaire renvoie à plusieurs modèles théoriques sur les déterminants du développement de l'enfant. L'étude CPE mesure les variations entre les caractéristiques de l'enfant et celles de son environnement familial et social, mais n'établit pas de relation de cause à effet entre les phénomènes observés. En fait, nous n'avons pas de données longitudinales qui permettraient de mesurer l'effet des expériences de vie à la petite enfance sur la préparation scolaire. De plus, il n'y a pas d'évaluation sur l'efficacité 
des programmes en petite enfance dans les quartiers visés. En conséquence, lors de l'appropriation des données de l'étude, les intervenants manquent encore d'information pour définir des priorités communes et revoir leurs plans d'action locaux. À Montréal, cette réflexion apparaît plus ardue encore puisque les intervenants agissent dans des quartiers comptant un grand nombre d'enfants à risque d'échec scolaire. Aussi, la plupart des administrations publiques, compte tenu des budgets disponibles, peuvent difficilement offrir davantage de services à leur communauté.

\section{Conclusion}

L'initiative Comprendre la petite enfance se poursuivra au cours des prochaines années. Lors du discours du trône en février 2004, le gouvernement fédéral affirmait sa volonté de créer 100 nouveaux sites du genre à travers le Canada.

Sur le territoire montréalais ciblé, le degré de préparation des jeunes à leur

Sur le territoire montréalais ciblé, le degré de préparation des jeunes à leur entrée à l'école varie selon les caractéristiques personnelles des enfants, mais aussi selon les conditions socioéconomiques de leur famille et de leur communauté. entrée à l'école varie selon les caractéristiques personnelles des enfants, mais aussi selon les conditions socioéconomiques de leur famille et de leur communauté. Les résultats montrent que dans un même quartier, les enfants éprouvent souvent des difficultés similaires, qui doivent être prises en compte dans l'élaboration et la révision des programmes locaux d'intervention précoce.

Comment la communauté peut-elle mobiliser davantage ses ressources pour aider les enfants à mieux se préparer à réussir à l'école? En comparaison avec les autres provinces canadiennes, le Québec est à l'avant-garde dans le domaine des services à l'enfance et à la famille. En effet, plusieurs politiques québécoises visent à assurer une meilleure préparation scolaire des enfants. Il apparaît donc prioritaire d'évaluer comment les programmes d'intervention issus de ces politiques contribuent effectivement au développement des enfants. Rappelons que les résultats de notre étude révèlent que les programmes de prématernelle en milieu scolaire ne permettent pas, à eux seuls, de réduire les écarts entre les populations d'enfants plus défavorisés et moins défavorisés.

L'initiative CPE montre l'importance de disposer d'indicateurs sur l'évolution des caractéristiques des enfants, des familles et des communautés. En ce sens, l'analyse longitudinale des trajectoires scolaires des enfants de la maternelle devrait nous aider à mieux comprendre comment certains d'entre eux parviennent à se développer pleinement, malgré qu'ils aient vécu dans un environnement moins favorable à leur épanouissement. 


\section{Références bibliographiques}

BRONFENBRENNER, U. et MORRIS, P.A. (1998). The ecology of development process. Dans W. Damon et R.M. Lerner (Eds.), Handbook of child psychology theoretical models of human development, $5^{\text {th }}$ edition (pp. 993-1029). New York, NY: John Wiley et Sons.

BROOKS-GUNN, J., DUNCAN, G. J. et ABER, L. J. (1997). Neighborhood poverty: Context and consequences for children. New York, NY: Russel Sage Foundation.

CONNORS, S., BRINK, S. et Développement des Ressources Humaines du Canada (1999). Comprendre la petite enfance: l'influence de la collectivité sur le développement de l'enfant. Ottawa, ON : Développement des ressources humaines du Canada.

Conseil des Ministres de l'Éducation (Canada) et Statistique Canada (2003). Indicateurs de l'éducation au Canada. Ottawa, $\mathrm{ON}$ : Rapport du programme d'indicateurs pancanadiens de l'éducation 2003.

Développement des Ressources Humaines du Canada et Statistique Canada (1996). Grandir au Canada: Enquête longitudinale nationale sur les enfants et les jeunes. Ottawa, ON : Statistique Canada.

Développement des Ressources Humaines du Canada et Statistique Canada (2003). Le bien-être des jeunes enfants au Canada. Rapport du gouvernement du Canada, Ottawa.

DOHERTY, G. et Développement des Ressources Humaines Canada (1997). Zero to six: The basis for school readiness. Ottawa, ON : Développement des ressources humaines Canada.

Early Years Action Group (2001). Early Years Action Group Getting in touch. Rapport produit par The Early Years Action Group, Toronto, North Quadrant.

JANUS, M. et OFFORD, D. (2000). Reporting on readiness to learn in Canada. ISUMA Canadian Journal of Policy Research, 1, 71-75.

KAGAN, S. L. et NEUMAN, M. J. (1997). Defining and Implementation school readiness: Challenges for families, early care and education, and school. Dans R. P. Weissberg, T. P. Gullotta, R. L. Hampton, B. A. Ryan et G. R. Adams (Eds.), Establishing preventive services healthy children 2010, Issues in children's and families lives Vol. 9 Thousand Oaks: Sage Publications, 61-96.

KOHEN, D. E., HERTZMAN, C., BROOKS-GUNN, J. et Développement des Ressources Humaines du Canada (1998). Les influences du quartier sur la maturité scolaire de l'enfant. Ottawa, ON : Développement des ressources humaines Canada.

KSI Research International Inc (2003). Comprendre la petite enfance: Développement de la petite enfance sur le territoire montréalais étudié (Québec). Ottawa, ON : Développement des ressources humaines Canada. 
LADD, G. W. (1996). Shifting ecologies during the 5-7 year period: Predicting children's adjustment during the transition to grade school. Dans A. Sameroff et M. Haith (Eds.), The five to seven year shift: The age of reason and responsibility (pp. 363-386). Chicago, IL: University of Chicago Press.

LA PARO, K. M. et PIANTA, R. C. (2000). Predicting children's competence in the early school years : A meta-analytic review. Review of educational research, 70(4), 443-484.

LAPOINTE, P., MARTIN, I. et ROBITAILLE, É. (2003). Le profil de cinq communautés montréalaises et le développement des enfants : Comprendre la petite enfance. Rapport présenté au ministère Développement des ressources humaines du Canada, Montréal, ISBN 2-921579-103.

LAPOINTE, P. et ROBITAILLE, É. (2002). Comprendre la petite enfance...à Montréal: nos enfants sont-ils bien préparés à commencer l'école? Brochure d'information, Montréal.

MAY, D. C. et KUNDERT, D. K. (1997). School readiness practices and children at-risk: Examining the issues, Psychology in school, 34, 73-84.

National Education Goals Panel (1995). National education goals report executive summary: Improving education through family-school community partnerships. Washington, DC: Nation Education Goals Panel.

PIANTA, R. C. et COX, M. J. (1999). The changing nature of the transition to schools: Trends for the next decade. Dans R. C. Pianta et M. J. Cox (Eds.), The transition to kindergarten (pp. 363-380). Baltimore, MD : Paul H. Brookes Publishing.

RAMEY, S. L. et RAMEY, C. T. (1994). The transition to school: Why the first few years matter for a lifetime. Phi delta kappan, Novembre, 194-199.

RIMM-KAUFMAN, S. E. et PIANTA, R. C. (2000). An ecological perspective on the transition to kindergarten: A theoretical framework to guide empirical research. Journal of Applied Developmental Psychology, 21(5), 491-511.

STIPEK, D. J. et RYAN, R. H. (1997). Economically disadvantaged preschoolers: Ready to learn but further to go. Developmental Psychology, 33(4), 711-723.

WILSON, W. J. (1987). The truly disadvantaged: The inner city, the underclass, and public policy. Chicago, IL: University of Chicago Press. 